\title{
Temperature compensated Radio-Frequency Harmonic Bulk Acoustic Resonators Pressure Sensors
}

\author{
T. Baron, E. Lebrasseur, J.P. Romand, \\ S.Alzuaga, S. Queste, G. Martin, D. Gachon, \\ T. Laroche, S. Ballandras \\ FEMTO-ST, Université de Franche-Comté, \\ ENSMM, CNRS, UTBM, \\ Besançon, France \\ Email: thomas.baron@femto-st.fr
}

\begin{abstract}
In this work, we propose a compensated temperature pressure sensor fabricated on compound $\mathrm{LiNbO}_{3} / \mathrm{Quartz} / \mathrm{Quartz}$ substrates obtained by $\mathrm{Au} / \mathrm{Au}$ bonding at room temperature and double face lapping/polishing of $\mathrm{LiNbO}_{3} /$ Quartz stack and a final gold bonding with a structured Quartz wafer. This paper shows the possibility to obtain device which is intrinsically low sensitive to thermal effects, and even allowing a second order compensation thanks to the Quartz thermal stability Sensitivity of the final sensor to bending moments then is tested and results show pressure sensitivity of such devices.
\end{abstract}

BAW, Pressure Sensor, HBAR, Temperature compensation

\section{INTRODUCTION}

Single crystal-based acoustic resonators for pressure sensors have received a strong interest for many years. Various developments have been particularly achieved using Quartz and $\mathrm{LiNbO}_{3}$ with either SAW delay lines or resonators. However, most approaches have been developed exploiting $\mathrm{LiNbO}_{3}$ machining along standard etching, rarely compatible with batch processes as used for MicroElectro-Mechanical Systems (MEMS). In this works, we propose a new fabrication based on bonding different wafers and lapping polishing it. This process allows us to control piezoelectric film properties (velocity, coupling temperature sensitivity, and so on). Then, we can address one other challenge of the radio-frequency bulk acoustic devices: the temperature stability of their resonance frequency.

The proposed HBAR are based on the conjugation of the strong coupling coefficient of a thinned piezoelectric film and of a high intrinsic quality of the propagation substrates. We use single crystal films obtained by grinding contrarily to deposition or Smart-Cut approaches as this approach reveals easier to develop than the other mentioned ones. Our solution allows us to choose various single crystal piezoelectric films and hence offers many degrees of freedom for resonator optimization. We present a HBAR combining lithium niobate and

\author{
J. Masson, S. Ballandras \\ SENSeOR SAS, \\ TEMIS Innovation, \\ Besançon, France
}

quartz, allowing for the excitation of temperature compensated shear waves at various frequencies.

The pressure sensor is based on BAW structure. BAW devices submitted to flexural bending do not significantly shift in frequency due to acceleration of the shear wave upper the neutral line of deformation and the deceleration of the shear wave under this line. The creation of a cavity near the neutral line allows only accelerating or decelerating the waves, yielding a frequency shift.

We based our device fabrication on gold bonding with a lapping/polishing process to prepare wafer compound to different devices [1]. This process allows a collective manufacturing of sensors contrarily to classical approaches. A gold thin layer $(200 \mathrm{~nm})$ is deposited first by sputtering on both $\mathrm{LiNbO}_{3}$ and quartz wafers. Both wafers then are bonded together via gold layer compression into an EVG bonding machine. During this process, we apply a pressure of $65 \mathrm{~N} . \mathrm{cm}^{-2}$ to the whole contact surface, yielding a high quality bond. $\mathrm{LiNbO}_{3}$ is subsequently thinned by lapping and polishing steps to an overall thickness of 100 microns. We finally seal the first stack with a structured quartz wafer to form cavity. Aluminum electrodes are deposited on the $\mathrm{LiNbO}_{3}$ upon the cavity to achieve working BAW resonators. Sensitivity of the final sensor to bending moments then is tested. Q factors in excess of 10000 above $1 \mathrm{GHz}$ have been obtained along this approach.

\section{SENSOR DESCRIPTION}

The fabrication of Surface Acoustic Wave (SAW) pressure sensor based on membrane was strongly developed due to the wave frequency dependence versus tensile stress at the surface of the membrane when bent by pressure. In the case of High-overtone Bulk Acoustic Resonator (HBAR) propagating in such a membrane, the strain variations across the membrane thickness forbid the use of such an approach to develop pressure sensor applications. This can be easily demonstrated using for instance static finite element analysis with a very 
simple mesh as illustrated in fig.1. Indeed, the strain and hence the stress change their signs along the membrane thickness, as shown in fig. 2. As a consequence, the strain variation across the HBAR generates equilibrated velocity variations. On the one hand, the strain below the membrane neutral line yields an increase of resonant frequency of the HBAR; on the other hand, the strain above the neutral line yields a decrease of this frequency. Consequently, the resulting frequency shift is negligible.

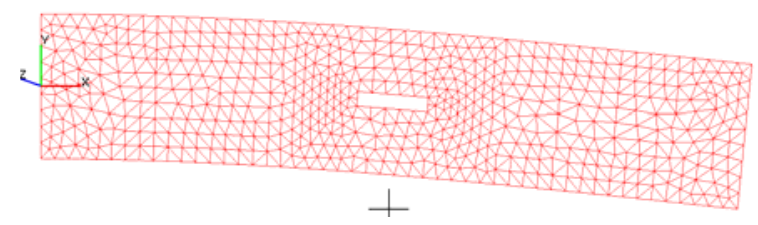

Figure 1. Cantilever beam submitted to bending force simulated by static finite element analysis. The defect in the middle of the beam is used further in the proposed development

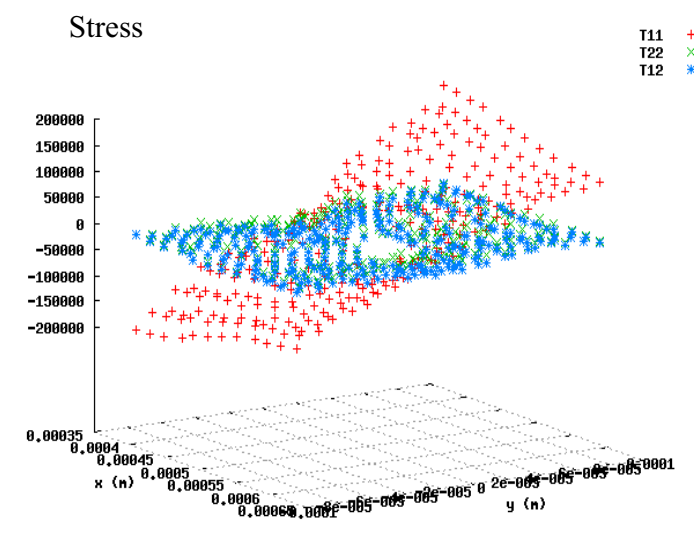

Figure 2.

Stress variations as a function of the crystallographic direction.

In the case of a cantilever beam, one can imagine to manufacture a micro-cavity near the neutral line, without changing fundamentally its location. If the transducer of the HBAR structure is straight above this micro-cavity, the emitted bulk waves are reflected by this micro-cavity and hence confined in this membrane location. The microcavity then plays the role of a mirror for the waves, as illustrated in fig.3. The structure of such device is shown in fig.4. First, a standard HBAR structure is built with the above-mentioned defect located straight under the transducer area. This structure then is bonded on a plane wafer, yielding the expected material stack. The surface of the cavity should at minimum coincide strictly to the surface of the transducer, but to ease the fabrication (particularly to manage alignment issues) the cavity largely overlaps the transducer aperture.

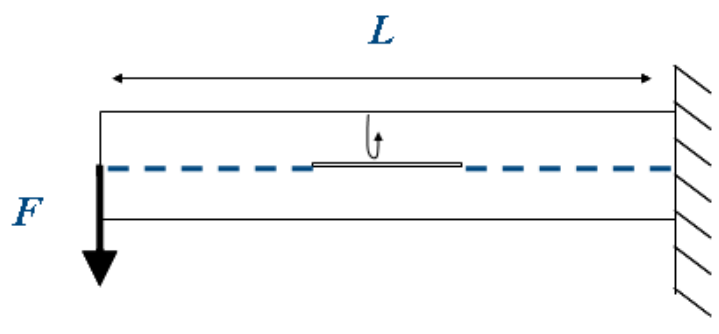

Figure 3. Waves and neutral line behaviors in the case of cantilever with micro-cavity and HBAR built above it.

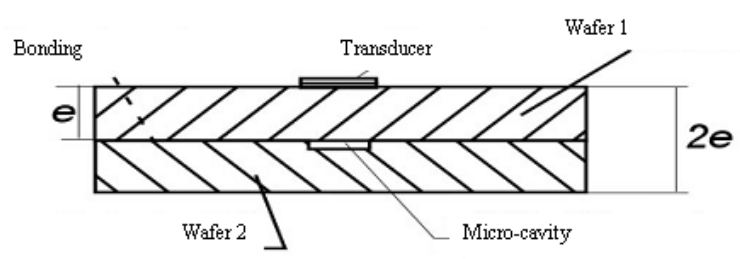

Figure 4. Archetypal structure of the device (the micro-cavity surface coincides at minimum with the transducer aperture)

As already mentioned, we combined a high piezoelectric coupling material for the fabrication of the HBAR transducer layer and a high acoustic quality material as propagation substrate. We then have chosen lithium niobate and quartz in that purpose. $\mathrm{LiNbO}_{3}$ actually exhibits crystal orientations for which pure shear wave can be excited with a coupling coefficient $\mathrm{K}_{\mathrm{s}}{ }^{2}$ in the vicinity of $50 \%$. The IEEE std- $176(\mathrm{YXl}) / 163^{\circ}$ cut is particularly well suited in that matter, as the coupling coefficients of the longitudinal mode drops to zero in that angular region whereas maximum shear wave coupling occurs simultaneously. It is associated to the AT cut of quartz which is wellknown for its second order temperature compensation of the slow shear mode together with high quality factors, yielding $Q . f$ products near $1.5 \times 10^{13}$ for standard BAW resonators (near 5 $\mathrm{MHz}$ ). However, a particular care must be taken when bonding one material on another, as they do not belong to the same class of symmetry. We then have to align the rotated $\mathrm{Z}$ axis of one wafer with $\mathrm{X}$ axis of the other to correctly transmit the wave according to polarization issues. Along this approach, it reveals theoretically using a standard anisotropic 1D model that zero temperature coefficients of frequency (TCF) can be obtained and optimized along the mode order. Also the use of the other temperature-compensated quartz orientations for BAW, and hence of any other material sharing such property, has been checked theoretically and reveals applicable as well. The next sections are devoted to the experimental validation of this concept, showing the possibility to compensate high frequency resonances in a similar way than what achieved at low frequencies $(5-15 \mathrm{MHz})$ with classical BAW resonators on quartz.

\section{PROCESS FLOW}

The process flow-chart reported in fig. 6 allows one for a collective manufacturing of the HBAR- 
based sensors previously described. As explained in Introduction, we use thinned single-crystal piezoelectric materials and Silicon combined with a room temperature $\mathrm{Au} / \mathrm{Au}$ bonding technique to achieve the elementary HBAR structure. As shown below, a supplementary bonding operation is required to manufacture the sensor. More details are reported hereafter to explain the implemented approach.
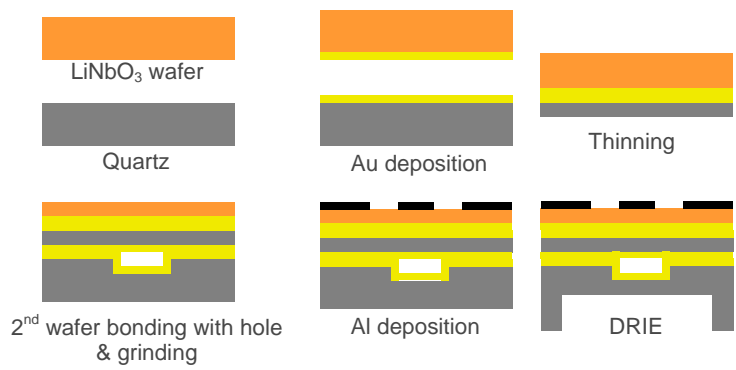

Figure 5. Process flow-chart for the fabrication of the HBAR pressure sensor

\section{A. Single-crystal wafer bonding and lapping/polishing}

The process we have implemented in this work has been already presented in previous publication. We then briefly recall here the basic principles and refer for detailed description to [2]. $\mathrm{A} \mathrm{LiNbO}_{3}$ wafer is bonded onto the Quartz substrate via the thermomechanical compression of the 200nm thick gold layers into an EVG wafer bonding machine as shown in fig.7. The bonding quality can be particularly controlled by adjusting the process duration and various parameters such as the applied pressure, the process temperature, the quality of the vacuum during the process, and so on. The bonding homogeneity is controlled using ultrasonics techniques.

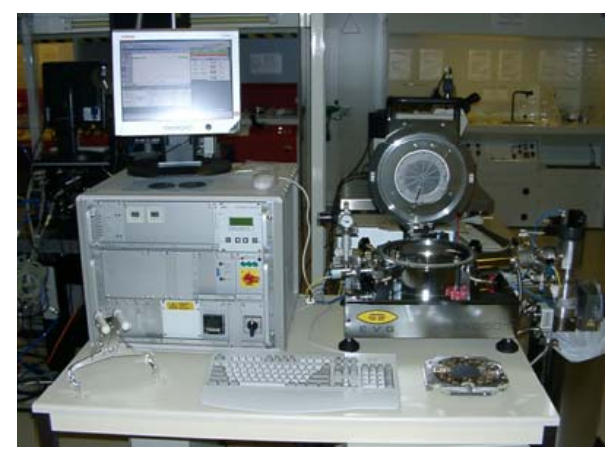

Figure 6.

Wafer Bonder EVG

The piezoelectric wafer is subsequently thinned by a lapping step to an overall thickness of 100 microns. The lapping machine used in that purpose and shown in fig.7 is a SOMOS double side lapping/polishing machine based on a planetary motion of the wafers (up to 4" diameter) to promote abrasion homogeneity. We use an abrasive solution of silicon carbide. We can control the lapping speed via rotation velocity, wafer load and abrasive concentration flow rate. It is then followed by a micro-polishing step using smaller abrasive grains.

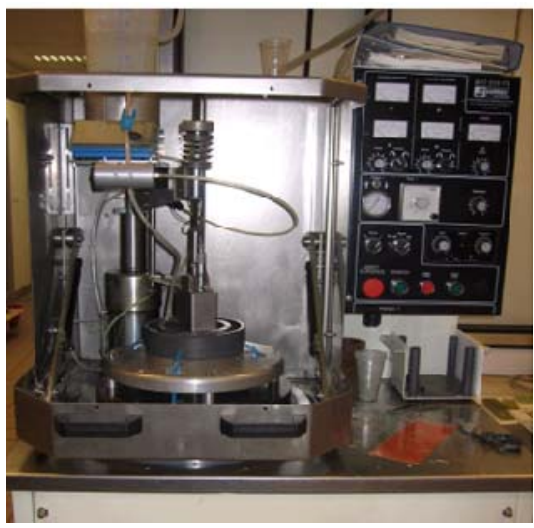

Figure 7.

SOMOS lapping/polishing machine

\section{B. HBAR fabrication}

Aluminum electrodes are deposited on the thinned $\mathrm{LiNbO}_{3}$ plate surface with a lift-off process. This top electrode allows for connecting the HBARbased sensor and for characterization operations.

\section{ELECTRICAL CHARACTERISATION}

We have tested first the principle of pressure sensor with a beam. A bench has been developed to apply different mass at the back of the HBAR sensor near the micro-cavity location. Figure 8 shows the bench first developed to characterize SAW sensitivity to stress effects. The device is wirebonding to SMA connector to plug it to our Rohde\&Schwarz ZVR network analyzer. Once connected, the sensor is loaded by metallic disks of calibrated mass and the reflection coefficient $S_{11}$ is recorded to characterize the device stress sensitivity.

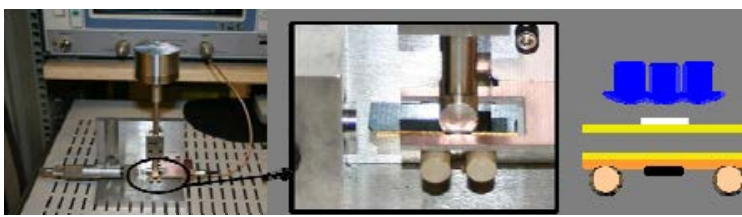

Figure 8. Photos and principle scheme of the mechanical bench for stress sensitivity characterization

Electrical responses are plotted versus applied mass in fig.9 showing a shift of the resonance frequency of the HBAR as expected.

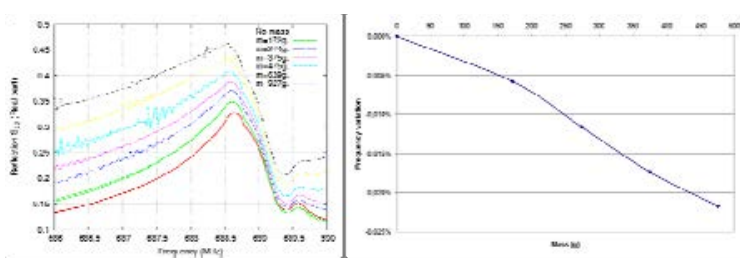

Figure 9.

Electrical results: $\mathrm{S}_{11}$ magnitude variations vs. applied mass.

We then use a test bench [3] which allows us to apply different pressures on the HBAR sensor near the micro-cavity location (fig. 10). The reflection 
coefficient $S_{11}$ then is recorded as previously to characterize the device pressure sensitivity. Figure 11 illustrates this experiment procedure and typical electrical responses are reported in fig. 12 .

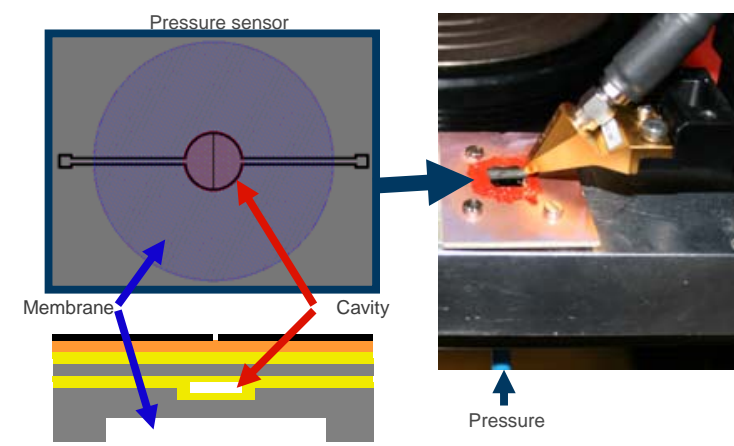

Figure 10. Photos and principle scheme of the mechanical bench for pressure sensitivity characterization

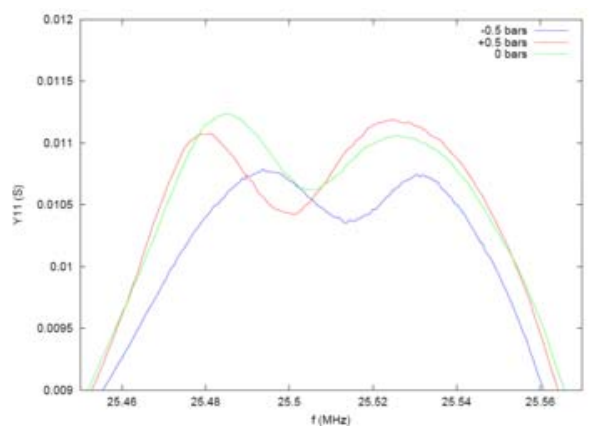

Figure 11. Electrical results: $\mathrm{Y}_{11}$ magnitude variations vs. applied pressure

The measured electrical responses show a shift of the resonance frequency of the HBAR versus pressure variations as expected. Further developments now must be dedicated to estimate theoretically the sensor ultimate sensitivity and to a quantitative theory/experiment assessment.

As our test bench is equipped with a thermal chuck, allowing us to apply different temperature on the HBAR, experimental characterization of TCF of different harmonics of the resonator has been achieved. Figure 13 shows frequency variation vs. temperature for four picks of resonance on one device. We can observe for the mode $\mathrm{C}$ the temperature compensation near room conditions $\left(\mathrm{T}_{\text {turnover }} \sim 50^{\circ} \mathrm{C}\right)$. We also observe a good agreement between the electrical results and our computation.

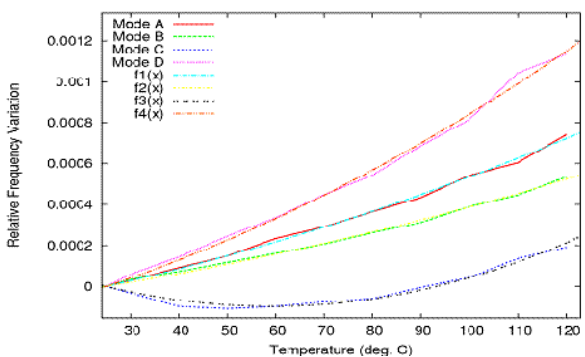

Figure 12. Frequency variation vs. temperature for different picks of resonance of one HBAR
When analyzing more closely one pick of resonance, we emphasize a quality factor of about 15000 at $1.274 \mathrm{GHz}$, as shown in fig.16. The Q.f products then is equal to $1.8810^{13}$ which overcomes the low frequency BAW values and is conform to the state of art with quartz-based HBAR devices.

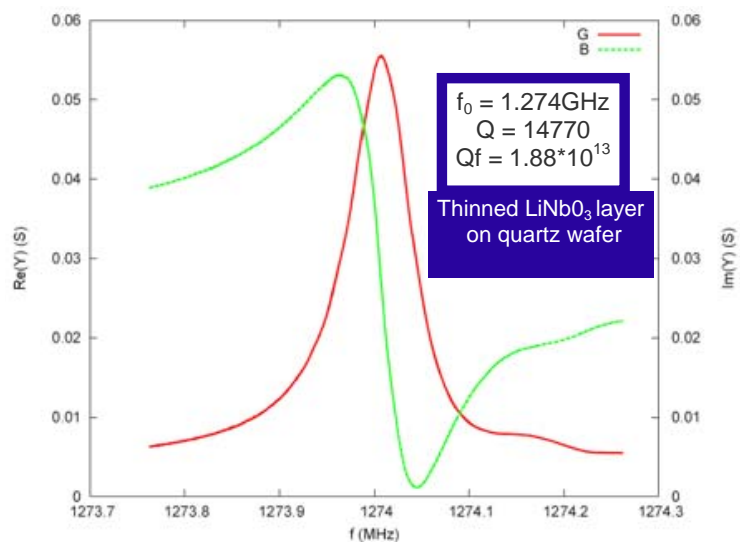

Figure 13. Electrical characterization of one pick of resonance of one HBAR and is Q.f products of $1.8810^{13}$.

\section{CONCLUSION}

In this paper, we have proposed new concepts for minimizing temperature sensitivity of HBAR devices exploiting single-crystal properties and to increase their stress sensibility for the development of pressure sensors. TCF near zero at room temperature have been observed for different modes, showing the relation between the mode order and the thermal compensation effect. Also the HBARbased pressure sensor concept has been experimentally validated. Optimization of such sensors is currently under development

\section{ACKNOWLEDGMENT}

This work has been supported by the French National Network for Research in Communication (RNRT) as the OASIS project under grant \#98 S 0324 and also by the Centre National d'Etudes Spatiales (CNES) under grant \#04/CNES/1941/00DCT094, and by the Direction Generale pour l'Armement (DGA) under grants \#05.34.016 and \#07-34-020.

\section{REFERENCES}

[1] D. Gachon, E. Courjon, G. Martin, L. Gauthier-Manuel, J.C. Jeannot, W. Daniau, S. Ballandras, "Fabrication of High Frequency Bulk Acoustic Wave Resonator Using Thinned Single-Crystal Lithium Niobate Layers", Ferroelectrics, vol. 362 , pp. $30-40,2008$

[2] T. Baron, D. Gachon, G. Martin, S. Alzuaga, D. Hermelin J.P. Romand, "Temperature Compensated RadioFrequency Harmonic Bulk Acoustic Resonators", proc. Of the 2010 IEEE International Frequency Control Symposium

[3] T. Baron, D. Hermelin, J.P. Romand, S. Alzuaga, S. queste, J.Y. Rauch, J.F. Leguen, B. Belgacem, S. Ballandras, "SAW pressure sensor on quartz membrane lapping", proc. Of the $10^{\text {th }}$ French Congress on Acoustics, Lyon, April 12-16, 2010 